\title{
Verhaltenstherapie
}

\section{Mitteilungen der Verbände / Information by Behavior Therapy Associations}

Verhaltenstherapie 2021;31:168-171

DOI: 10.1159/000516541

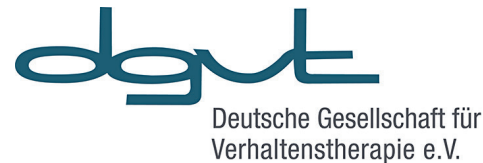

\section{Deutsche Gesellschaft für Verhaltenstherapie (DGVT) e.V.}

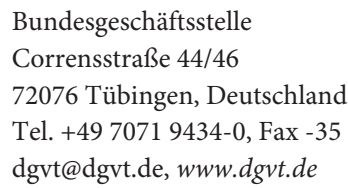

\section{Ratgeber Depression als Hilfestellung für alte Menschen mit Pflegebedarf}

Dieser Ratgeber richtet sich an von Depression Betroffene sowie deren Vertraute und Angehörige und geht der Frage nach, woran man Depression bei Pflegebedarf im Alter erkennt - bei sich selbst und beim anderen. Sie finden darin Anregungen, wie Sie als von Depression Betroffene im alltäglichen Leben mit Depression umgehen können, sowie Tipps und Ratschläge dazu, was Vertraute und Angehörige tun können, um Betroffene zu unterstützen.

Eine Print-Version des Ratgebers kann über den DGVT-Verlag bestellt werden. Der Ratgeber steht auch als PDF-Dokument sowie als Audiodatei auf unserer Homepage ( $w w w . d g v t . d e)$ zur Verfügung.

Wir freuen uns, wenn Sie Interesse an dem Ratgeber haben und diesen selbst in Ihrem beruflichen Kontext nutzen oder an Kolleg*innen, Pflegekräfte, an Patient*innen oder Angehörige von Patient ${ }^{\star}$ innen weiterreichen.

Entstanden ist der Ratgeber im Rahmen des Projektes PSY-CARE, welches die DGVT und ihr Berufsverband unterstützt. Mehr Informationen zum Projekt PSY-CARE finden Sie hier: https://psy-care.de/

Der Ratgeber darf nicht zu kommerziellen Zwecken genutzt und weitergegeben werden. Sollten Sie Interesse haben, den Ratgeber (Digital-, Print- oder Audio-Version) auf Ihrer Homepage zu veröffentlichen, kontaktieren Sie bitte die Autorin (eva-marie.kessler@medicalschoolberlin.de). Falls Sie eine umfangreichere Nutzung des Werks anstreben, kontaktieren Sie bitte ebenfalls die Autorin.

\section{Fort- und Weiterbildung}

In Kooperation mit der Deutschen Gesellschaft für Systemische Therapie, Beratung und Familientherapie (DGSF) und der Systemischen Gesellschaft (SG) wird die DGVT e. V. eine Online-Fachtagung zum Thema „Systemische Therapie meets Verhaltenstherapie - an evolving affair: ,Ich sehe was, was Du nicht siehst ..." vom 15. - 16. Oktober 2021 veranstalten. Das Tagungsprogramm setzt sich aus Expert*innen-Impulsvorträgen, Live-Demonstration, Workshops und einer interaktiven Reflexion zusammen.

Die 20. DGVT-Praxistage der Kinder- und Jugendlichenpsychotherapie mit dem Titel „Von A wie Angst bis $Z$ wie Zeugnis - Schule meets Psychotherapie" finden am 27. November 2021 online über Zoom statt. Im Rahmen von Kurz- und Langworkshops befassen sich Teilnehmer ${ }^{*}$ innen mit Inhalten rund um das Thema Psychotherapie und Schule.

Ausführliche Informationen zu diesen und weiteren Angeboten erhalten Sie unter www.dgvt-fortbildung.de oder über die DGVT-Bundesgeschäftsstelle in Tübingen, Tel.: 070719434 34, Fax: 070719434 35, E-Mail: fortbildung@dgvt.de. Sprechzeiten: Di und Do 9:00-12:00 Uhr; Di und Mi 14:00-15:30 Uhr.

DGVM

Deutsche Gesellschaft für Verhaltensmedizin und Verhaltensmodifikation e.V.

Deutsche Gesellschaft für Verhaltensmedizin und Verhaltensmodifikation (DGVM)

Prof. Dr. Thomas Kubiak

Psychologisches Institut

Abteilung Gesundheitspsychologie

Johannes Gutenberg-Universität Mainz

Binger Straße 14-16

55122 Mainz, Deutschland

\section{Neue Mitglieder:}

Wir begrüßen herzlich unser neues Mitglied:

Dr. Marco Warth (Heidelberg) 


\section{Aus dem Vorstand}

Die Preisträgerinnen der aktuellen Runde des Peer Mentoring Programms stehen fest. Aus den eingegangenen Anträgen wurden folgende fünf Bewerbungen zur Förderung ausgewählt:

- Dr. Jana Strahler (Gießen) und Dr. Julia Reichenberger (Salzburg)

- Dr. Nora Schaal (Düsseldorf) und Dr. Rita Amiel Castro Meyer (Zürich)

- Dr. Anna Pohl (Köln) und Laura Klewinghaus (Wuppertal)

- Rebekka Büscher (Freiburg) und Lena Steubl (Ulm)

- Katharina van Stein (Heidelberg) und Kathrin Schubert (Marburg)

\section{Herzlichen Glückwunsch!}

Im Oktober (07.-09.10.2021) wird ein speziell auf Nachwuchswissenschaftlerinnen und Nachwuchswissenschaftler zugeschnittener virtueller Kongress, das 1. DGVM Early Career Researchers Event, stattfinden. Herr Prof. Dr. Hermann-Lingen (Göttingen) und Frau Prof. Dr. Ditzen (Heidelberg) konnten bereits für Beiträge $\mathrm{zu}$ „hot topics“ der Verhaltensmedizin gewonnen werden. Weiters sind die Vorstellung und Diskussion von Promotionsvorhaben und Workshops geplant. Die Teilnahme ist für Nachwuchswissenschaftlerinnen und Nachwuchswissenschaftler der DGVM kostenfrei.

\section{Internationales}

Der 16. Kongress der International Society of Behavioral Medicine (ISBM) wird vom 07.-11.06.2021 virtuell stattfinden. Das volle Programm ist hier einsehbar: https://www.delegate-reg.co.uk/icbm2021/programme.

Die International Association of Applied Psychology (IAAP) bietet am 16.10.2021 einen virtuellen Kongress als Early Career Marathon zum Thema "Integrating Research and Practice, 24 hours of Applied Psychology from Around the World" an, bei denen Projekte von Nachwuchswissenschaftlerinnen und -wissenschaftlern mit internationalen Expertinnen und Experten diskutiert werden können. Weitere Informationen dazu finden Sie hier: https://iaapsy.org/meetings/early-career-marathonintegrating-research-and-practicel

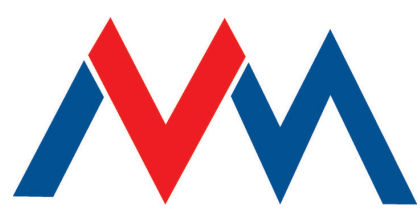

\section{AVM Österreich}

Arbeitsgemeinschaft für Verhaltensmodifikation (AVM) Büro Salzburg

Paris-Lodron-Straße 32, 5020 Salzburg, Österreich

Tel. +43662884166

Büro Wien

Leidesdorfgasse 11-13/Stiege 1/Top 8, 1190 Wien, Österreich

Tel. +4313204132

office@institut-avm.at, https://institut-avm.at

\section{WISSENSCHAFTLICHE TAGUNG DER AVM PERSÖNLICHKEITSSTÖRUNGEN \\ Stift Göttweig/Niederösterreich, 14. bis 17. Oktober 2021}

Persönlichkeitsstörungen (PS) sind und bleiben ein relevantes Thema weit über das Fachgebiet der Psychotherapie hinaus: So fanden sie etwa in der Ära Trump breiten medialen Niederschlag, um politische Phänomene pathopsychologisch zu deuten, und auch im Alltagsdiskurs werden Konzepte wie beispielsweise „narzisstische Mütter" aufgegriffen, um das Verhalten unserer Mitmenschen zu verstehen.

Aufgrund der Vielfältigkeit von PS fällt es schwer, die Auswirkungen auf das Leben der Betroffenen allgemein zu charakterisieren. Es besteht eine große Bandbreite beginnend mit einer erhaltenen sozialen Funktionsfähigkeit mit spür-, aber nicht immer zuordenbarem Leidensdruck bis hin zu einer vollständigen sozialen, psychischen und physischen Dekompensation, die bis zum Tod führen kann. Klassische Merkmale sind der häufig frühe Beginn, die Chronizität und die Neigung zu Komorbiditäten, die als Folge der erhöhten Vulnerablität und fehlschlagender Anpassungsversuche verstanden werden können.

Mit Prävalenzraten im Bereich von 10-15 \% (Fiedler, 2016, S. 209) stellen Menschen mit PS jedenfalls keine obskure Randgruppe, sondern einen im wahrsten Sinne des Wortes beachtenswerten Teil der Bevölkerung dar und begegnen uns somit nicht nur als Patient*innen, sondern ebenso im Alltagsleben, ob als Zufallsbegegnung, am Arbeitsplatz oder als Menschen, die uns nahe stehen.

Betroffenen eilt häufig der Ruf voraus, „schwierige“ Patient*innen zu sein: Die Beziehungsgestaltung soll sich herausfordernd gestalten, Fortschritte würden länger benötigen und die häufigen Komorbiditäten die Behandlung weiter verkomplizieren. Dementsprechend berichten Betroffene oft über Schwierigkeiten bei der Suche nach kompetenten und willigen Behandler*innen. Aller- 
dings lässt sich feststellen, dass Patient*innen mit PS in Erhebungen mit typischerweise 40-50 \% (Fiedler, 2016, S. 211f) ohnehin in einer dermaßen beeindruckenden Häufigkeit in ambulanten und stationären Settings vertreten sind, dass sich die Frage danach, ob jemand PS behandelt, eigentlich gar nicht stellt. Bezüglich der angemesseneren Frage, wie mit Menschen mit PS gearbeitet wird, weist gerade die Verhaltenstherapie mit der Dialektischen Verhaltenstherapie (DBT) und der Schematherapie attraktive, evidenzbasierte und in kontinuierlicher Weiterentwicklung befindliche Angebote auf, die auch in der Praxis zunehmend angenommen werden und klare Handlungsanweisungen für Beziehungsaufbau und komplexe Behandlungskonzepte zu geben vermögen. Das wird auch durch die Veröffentlichung der aktualisierten S3-Leitlinie zur Borderline-PS bestätigt (Lieb et al., 2020), in der erneut das Primat störungsspezifischer verhaltenstherapeutischer (und psychodynamischer) Ansätze in der Behandlung betont wird.

Für den Bereich der Diagnostik kann guten Gewissens von einer Revolution gesprochen werden, die das DSM-5 bereits zaghaft andeutete und nun durch die ICD-11 vollendet wird: Die Neukonzeptualisierung von ehemals acht möglichen Varianten zur Beschreibung gestörter Persönlichkeiten als multidimensionales deskriptives System vermag Therapieforschung als auch Therapiepraxis gleichermaßen $\mathrm{zu}$ stimulieren und besitzt eine erfreuliche Anschlussfähigkeit an verhaltenstherapeutische diagnostische Traditionen. Sie dürfte wohl auch bei unseren Patient*innen auf eine größere Akzeptanz stoßen als der bisherige Reduktionismus. Als Wermutstropfen verbleibt der Abschied von vertrauten und oft griffigen diagnostischen Schemata.

Indirekt aber von nicht zu unterschätzender Bedeutung für das Feld der PS ist die Aufnahme der komplexen posttraumatischen Belastungsstörung in die ICD-11. Die diagnostischen Kriterien der Defizite in der Affektregulation, im Selbstkonzept und in der Beziehungsgestaltung lassen ein gewisses Überschneidungspotential mit dem Konzept der PS erahnen. Angesichts der hohen Komorbiditätsrate mit posttraumatischen Belastungsstörungen und der Relevanz von Beziehungstraumata für die Entstehung von PS verwundert es nicht, dass erfolgreiche Behandlungsansätze auch eine traumatherapeutische Komponente aufweisen (z. B. Imagery Rescripting and Reprocessing Therapy - IRRT, in der Schematherapie und DBT-PTBS). Es bleibt zu hoffen, dass eine Integration der unterschiedlichen Perspektiven gelingen wird, sodass wechselseitige Anregung und Ergänzung und nicht etwa Konkurrenz und Doppelgleisigkeit die Folge sein werden.

Wir freuen uns darüber, dass es uns gelungen ist, mit unserem Programm diese bedeutsamen Themen aufzugreifen und dazu namhafte Expert*innen auf diesen $\mathrm{Ge}$ - bieten zu gewinnen. So werden im Rahmen der Tagung PS über die gesamte menschliche Entwicklungsspanne und unter Einbeziehung wichtigerer Lebensthemen wie Glück oder Gewalt thematisiert. Als besonders für PS geeignete verhaltenstherapeutische Behandlungsansätze sind die Dialektische Verhaltenstherapie und die Schematherapie vertreten. Die Neuerungen im Zuge der ICD-11 werden ebenso disktuiert wie auch der Überschneidungsbereich Traumatherapie und PS, der mit DBT-PTBS und IRRT präsent ist. Dabei ist es uns ein besonderes Anliegen, wichtige neue Entwicklungen anzusprechen, ohne Altbewährtes in Vergessenheit geraten zu lassen.

Herr Dr. Matías Valente (Schwäbisch Hall) eröffnet mit seinem Vortrag zur Schematherapie die Tagung: „Schematherapie im Kontext: ein Verfahren der ,3. Welle?". Mit seinem Workshop „Interpersonelle Konflikte und Intimitätsstörungen in der Schematherapie: von der Einzel-zur Paartherapie“ geht er ausführlich auf einen zentralen Problembereich der PS ein.

Herr Prof. Dr. Peter Fiedler (Heidelberg) widmet sich den Veränderungen im ICD-11 mit dem Vortrag: „Integrative Behandlung von Persönlichkeitsstörungen im Lichte der ICD-11". Weiters gestaltet er einen Workshop zur Bedeutung des zukünftigen Domain-Konzeptes in der ICD-11 für Diagnostik und Behandlung von Persönlichkeitsstörungen.

Anlässlich des Aufbaus einer störungsspezifischen Behandlungseinrichtung wird Herr Prof. Dr. Martin Bohus (Mannheim) unter dem Titel „DBT für komplexe PTSD im österreichischen stationären Setting " zunächst den theoretischen Hintergrund sowie den Stand der Forschung diskutieren, wonach Frau Mag. Romy Busch, Frau Elke Balt und Frau Mag. Marie-Luise Gerschein (alle Klinik Eggenburg) die praktische Implementierung beschreiben. Eine Vertiefungsmöglichkeit bietet der gleichnamige Workshop.

Seinen Tätigkeitsschwerpunkt Persönlichkeit und Straftäter wird Herr Mag. Wolfgang Marx (Wien) mit dem Vortrag „Und bist du nicht willig, so brauch' ich Gewalt - häusliche Gewalttaten und der Einfluss von Persönlichkeitsmerkmalen in heterosexuellen Intimpartnerschaften" vorstellen.

Den Themenbereich PS über die Lebensspanne eröffnet Frau Dipl.-Psych. Cornelia Hartung mit ihrem Vortrag „Die Diagnose der Persönlichkeitsstörung im Kindesund Jugendalter - Chance und/oder Stigma?", zudem wird es mit ihrem gleichlautenden Workshop die Möglichkeit geben, sich intensiver mit dem Thema auseinanderzusetzen.

Dr. Meinolf Peters (Marburg) führt die Auseinandersetzung mit dem Entwicklungsaspekt in seinem Vortrag „Persönlichkeitsstörungen im Alter - ein Thema für die Psychotherapie?" fort und ergänzt sie weiter durch seinen 
Workshop „Welche Bedeutung haben strukturelle Defizite im Alter?".

Herr Prof. Dr. Mervyn Schmucker (Dresden) und Frau Dipl.-Psych. Ines Riedeburg-Tröscher (Wiesbaden) runden die Tagung ab und halten zum Thema IRRT (Imagery Rescripting and Reprocessing Therapy) den Vortrag „Innere-Kind Therapie mit der IRRT - Der Ort an dem du das Glück erleben kannst!", was sie im dazugehörigen Workshop weiter ausführen.

Die AVM Österreich würde sich freuen, Sie bei der Tagung 2021 in Niederösterreich begrüßen zu dürfen.

Mag. Julia Kober und MMag. Daniel Weigl

(Leiterin und Stellvertreter des AK Niederösterreich der AVM)

\section{Anmeldung und Informationen}

Arbeitsgemeinschaft für Verhaltensmodiffikation (AVM Österreich)

Büro Salzburg/Tagungsbüro: Paris-Lodron-Straße 32, 5020 Salzburg, Österreich

Büro Wien: Leidesdorfgasse 11-13/Stiege 1/Top 8, 1190 Wien, Österreich

Telefon: $+43(0) 662 / 884166$

Fax: +43(0)662/88 6566

E-Mail: office@institut-avm.at

Home: https://institut-avm.at

Weitere Informationen zur Tagung unter https://institut-avm.at/tagung/23-wissenschaftliche-tagung-

persoenlichkeitsstoerungen/

\section{Literatur}

Fiedler, P., Herpetz, S. C. (2016). Persönlichkeitsstörungen. Weinheim, Basel: Beltz.

Lieb, K., Stoffers-Winterling, J. \& die Leitliniengruppe (2020). Die neuen S3-Leitlinien: Borderline Persönlichkeitsstörung. Psychotherapie Forum 24, 87-88. 